\title{
INFLUENCE OF GEOMETRICAL PROPERTIES FOR THE CALCULATION OF A PRESSURE-FREE WHOLE HEART GEOMETRY
}

\author{
Jochen Brenneisen $^{1}$, Steffen Schuler ${ }^{1}$, Ekaterina Kovacheva ${ }^{1}$, \\ Tobias Gerach $^{1}$, Olaf Dössel ${ }^{1}$ and Axel Loewe ${ }^{1}$ \\ ${ }^{1}$ Institute of Biomedical Engineering, Karlsruhe Institute of Technology (KIT) \\ Kaiserstraße 12, 76131 Karlsruhe \\ publications@ibt.kit.edu,www.ibt.kit.edu
}

Key words: Cardiac Modeling, Mechanic Simulation, Pressure-Free State, Unloaded Geometry

\begin{abstract}
Individualized computer models of the geometry of the human heart are often based on magnetic resonance images (MRI) or computed tomography (CT) scans. The stress distribution in the imaged state cannot be measured but needs to be estimated from the segmented geometry, e.g. by an iterative algorithm. As the convergence of this algorithm depends on different geometrical conditions, we systematically studied their influence. Beside various shape alterations, we investigated the chamber volume, as well as the effect of material parameters. We found a marked influence of passive material parameters: increasing the model stiffness by a factor of ten halved the residual norm in the first iteration. Flat and concave areas led to a reduced robustness and convergence rate of the unloading algorithm. With this study, the geometric effects and modeling aspects governing the unloading algorithm's convergence are identified and can be used as a basis for further improvement.
\end{abstract}

\section{INTRODUCTION}

Computer simulations of personalized models are of increasing significance in 21 st century medical research as they offer time- and cost-effective opportunities e.g. for therapy planning and outcome prediction. To resemble patient specific cardiac anatomy as precisely as possible, models are based on medical imaging data. Usually whole heart image stacks are acquired in end-diastatic state using MRI or CT techniques. Although blood pressure and tissue stress are minimal in this state, they are not zero and cannot be extracted from the image. Thus, a pressure-free state has to be reconstructed as a reference condition for simulations. Under the assumption that the pressure-free state corresponds to the stress-free state, several algorithms can be applied to calculate the pressure-free whole heart geometry. One of the simplest options among more complicated algorithms [1,2] is the backward displacement method [3, 4]. This iterative procedure is explained in detail in section 2.3. Based on the algorithm's results, the stress distribution in end-diastolic state can be estimated.

However, we and others [5] observed that the convergence of the unloading algorithm varied across different simulation setups and a robust determination of the unloaded geometry cannot be ensured. Thus, this work aims to identify potential error sources as a basis for increasing algorithm robustness. Hence, we systematically studied the influence of different geometrical and tissue properties on the convergence of this unloading algorithm. 

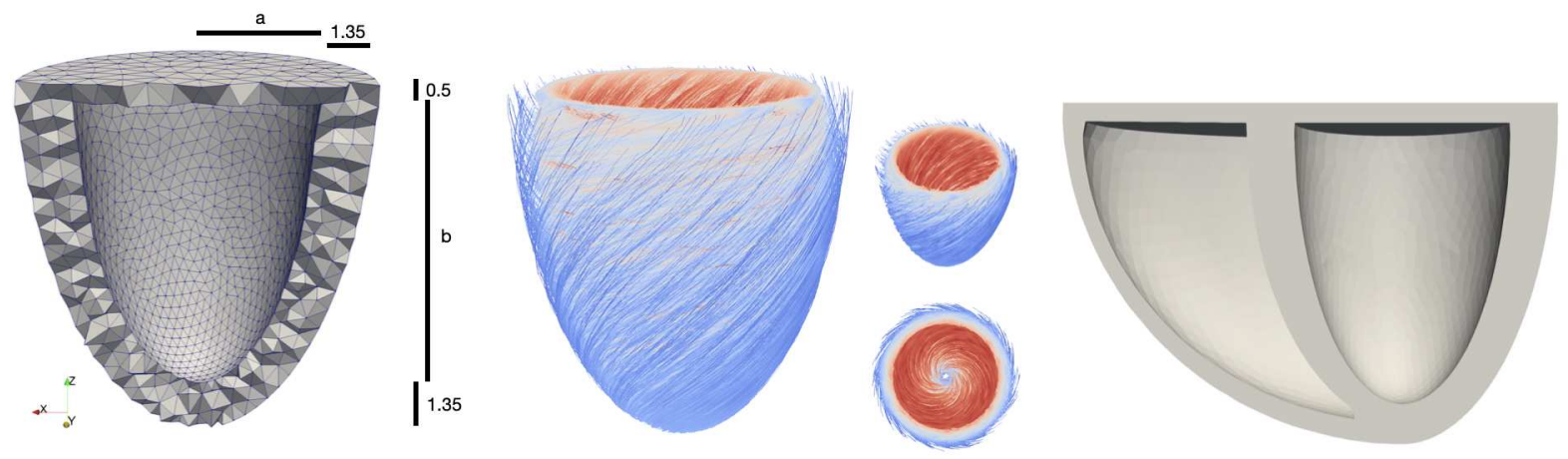

Figure 1: Idealized geometries cut in frontal plane. Left: LV with indication of relevant measures in millimeter. Wall and lid thickness were kept constant throughout all simulations. For short axis $a$ and long axis $b$ dimensions, see table 1. Middle: Fiber distribution for idealized LV cut off just below the lid. Fibers depicted as streamlines with fiber angle colour coded. Frontal, top and tilted view. Right: Biventricular geometry cut in frontal plane. Dimensions of LV identical to truncated sphere on the left.

\section{METHODS}

We first summarize the creation of the finite element method (FEM) models and present the corresponding material law. After the unloading algorithm itself is presented, four simulation scenarios are deduced.

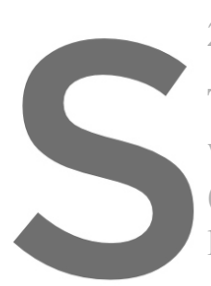

\subsection{Geometry creation}

To study the influence of

was split in different com

(LV) was represented by

left). A long axis length 0
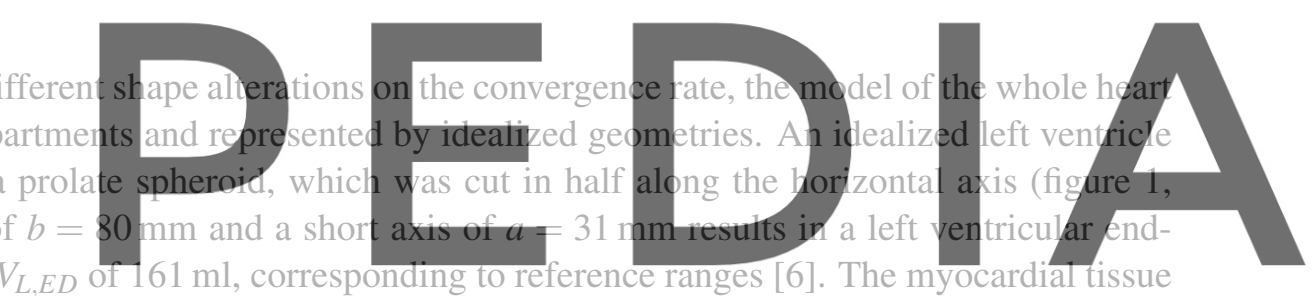

diastolic chamber volume $V_{L, E D}$ of $61 \mathrm{ml}$, corresponding to reference ranges [6]. The myocardiai tissue

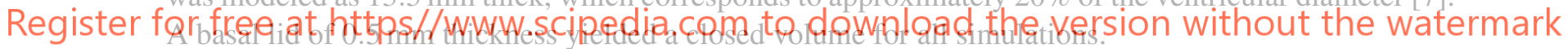

As the LV does not include concave areas, a biventricular model was implemented following Bakir et

al. [8]. Starting from the model of the LV, an additional eliiptical spheroid was added to represent the right ventricular (RV) endo- and epicardial walls (figure 1, right).

\subsection{Material properties}

The myocardial tissue of both ventricular geometries was modelled as a hyperelastic nearly incompressible material with the constitutive relation introduced by Guccione et al. [9]. The strain-energy function $W$ follows equation (1) with indices for fiber direction $f$, sheet direction $s$ and sheet normal direction $n$ and four constants $C, b_{f f}, b_{x x}$ and $b_{f x}$. Incompressibility was enforced with a penalty formulation related to the Jacobian of the deformation gradient $J=\operatorname{det} \mathbf{F}$ scaled by a bulk modulus $\kappa \gg 0 \mathrm{kPa}$.

$$
\begin{aligned}
W & =\frac{C}{2}\left(e^{Q}-1\right)+\frac{\kappa}{2}(J-1)^{2} \\
Q & =b_{f f} E_{f f}^{2}+b_{x x}\left(E_{n n}^{2}+E_{s s}^{2}+E_{n s}^{2}+E_{s n}^{2}\right)+b_{f x}\left(E_{f n}^{2}+E_{n f}^{2}+E_{f s}^{2}+E_{s f}^{2}\right)
\end{aligned}
$$

We used $C=162.78, b_{f f}=11.01, b_{x x}=4.4$ and $b_{f x}=7.71$ as standard values. 
Fiber directions were assigned by the Laplace Dirichlet Rule-Based (LDRB) algorithm proposed by Bayer et al. [10]. We used fiber angles of $+60^{\circ}$ on the endocardial wall and $-60^{\circ}$ on the epicardial wall. The resulting fiber distribution for the LV geometry is shown in the middle panel of figure 1.

\subsection{Unloading procedure}

To calculate the unloaded heart geometry, the iterative algorithm suggested by Bols et al. [4] was implemented (figure 2). We start with an initial geometry, e.g. a 3D FEM model created based on a segmentation of an MRI image stack. Thus, the non-inflated state is initialized with the MRI-derived inflated state, i.e.: $\mathbf{X}_{0}=\mathbf{x}_{m}$. In each iteration $i$, the geometry is inflated by applying a linearly increasing pressure on the endocardial surface of the concerning heart chamber over a time interval of $1 \mathrm{~s}$. For the LV, a pressure increase from 0 to $8 \mathrm{mmHg}$ was chosen [11], while the RV enddiastolic pressure is assumed to be $\approx 50 \%$ of the LV end-diastolic pressure [12] (in our case $p_{R V, E D}=4 \mathrm{mmHg}$ ). After the inflation, the residual vector $\mathbf{r}_{i}$ is calculated as the difference between the inflated state $\mathbf{x}_{i}$ and the initial geometry $\mathbf{x}_{m}$. Subsequently, the residual vector is applied as a backward displacement to the noninflated state $\mathbf{X}_{i}$ :
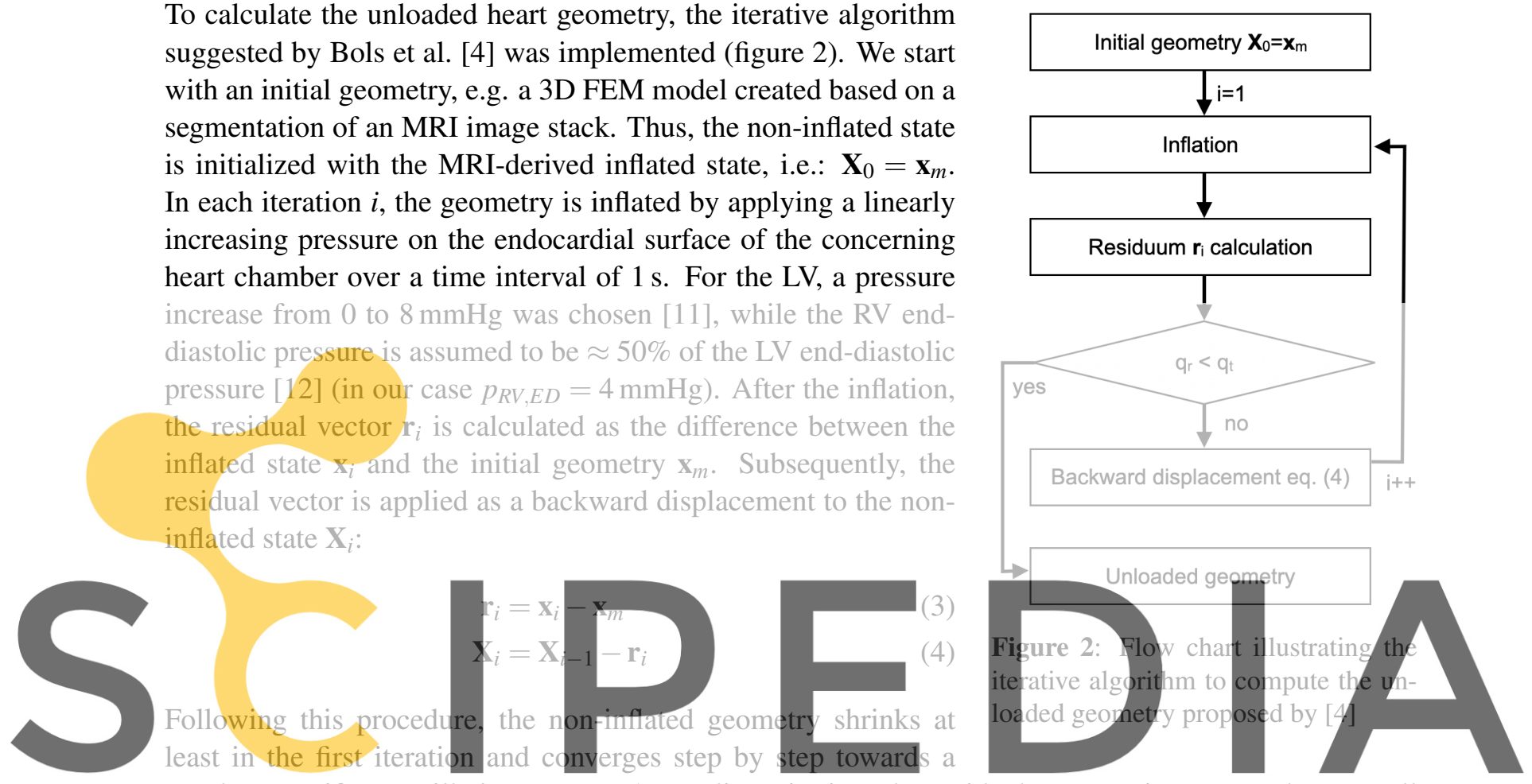
steady state, if no oscillations occur. As quality criterion, the residual norm $q_{r}$ is computed across all Register fordfree at https//www.scipedia.com to download the version without the watermark

$$
q_{r}=\|\mathbf{r}\|_{1}
$$

As long as $q_{r}$ does not fall below the threshold of $q_{t}=10^{-6} \mathrm{~m}$, the algorithm continues to iterate. Technically it has to be considered that the algorithm consists of fixed-point iterations, which are only guaranteed to converge, if they represent a contraction.

\subsection{Simulation Scenarios}

In this section, four simulation scenarios are deduced, covering the major influencing factors. All simulations were performed using CardioMechanics [13].

\subsubsection{Shape of the geometry}

Heart shape and curvature vary considerably between patients. In this scenario, we distinguished between flat areas (no curvature), as well as convex and concave shapes. While flat areas could easily be evaluated using the LV geometry, concave regions were only represented in the biventricular model. To additionally 
quantify the influence of regions with different curvature, the shape of the LV was altered by adapting the ratio of short to long axis $\frac{b}{a}$ like in table 1 .

Table 1: Variation of geometry curvature: Ratio between short and long axis

\begin{tabular}{cccc}
\hline & low curvature & standard & high curvature \\
\hline Short axis a $[\mathrm{mm}]$ & 28 & 31 & 34 \\
\hline Long axis b $[\mathrm{mm}]$ & 98 & 80 & 66 \\
\hline ratio $b / a$ & 3.5 & 2.58 & 1.94 \\
\hline
\end{tabular}

The ratios are chosen the way, that all geometries are convex, but curvature varies from a lower to a higher value. Volume and wall thickness were kept constant for the sake of comparability. The resulting geometries for the ratios in table 1 are shown in figure 3.

\subsubsection{Chamber volume}

The LV end-diastolic volume was varied by $\pm 37 \%$ (101 to $222 \mathrm{ml}$ ) to cover standard ranges for men and women [6].

\subsubsection{Degrees of freedom}

In this study, the tetrahedral finite elements are represented by linear shape functions (4 node tetrahedra, P1) by default. Fiber

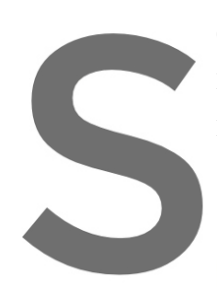

is known to suffer from mations. While a certain degree low curvature
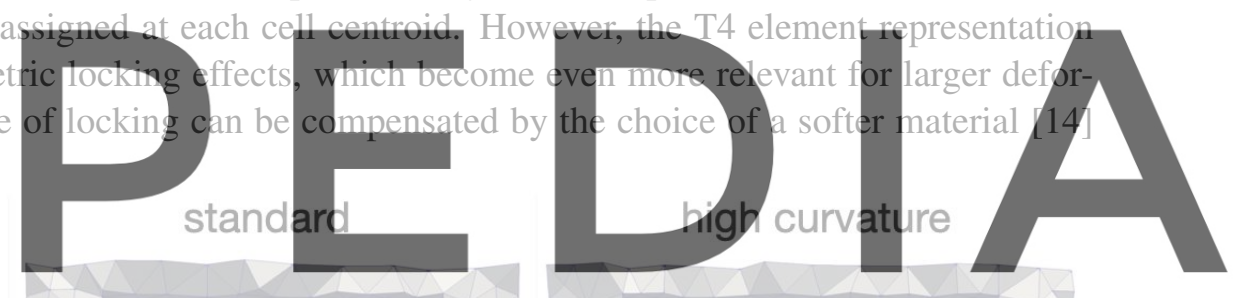

Register for free at https//www.scipedia.com to download the version without the watermark
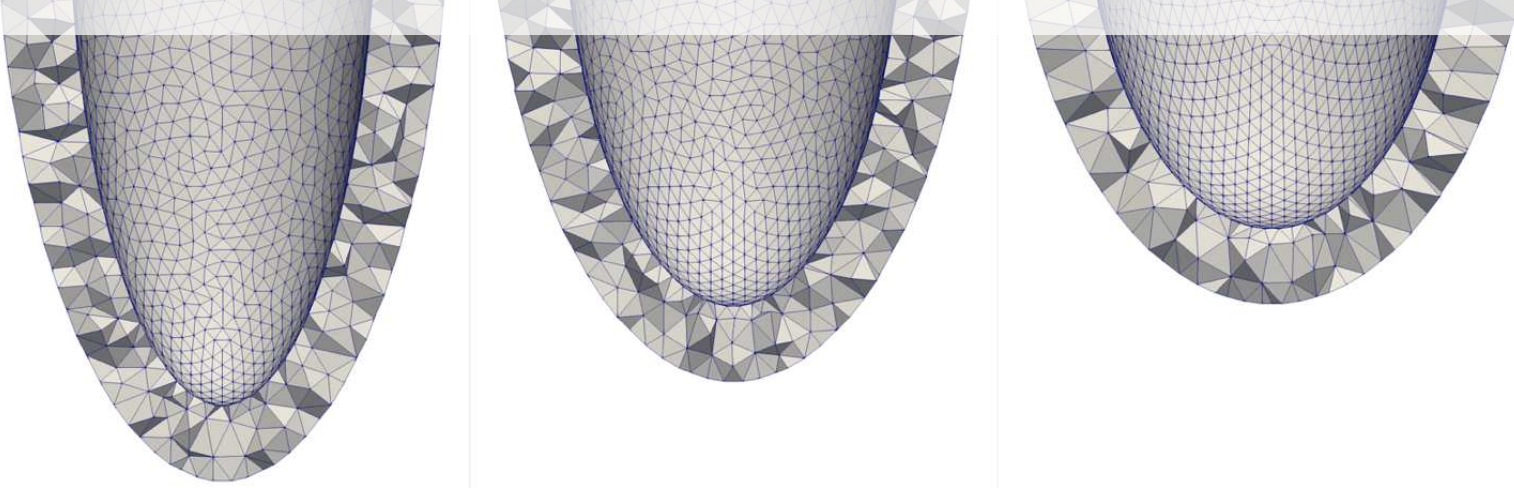

Figure 3: Variation of LV curvature in the order of table 1. The altered short to long axis ratio leads to different curvature. Left: Longer long axis, less convex shape. Middle: Standard ratio. Right: Shorter long axis, more convex shape. 
(see also following section), the volumetric locking effects can be strongly decreased by considering quadratic shape functions. These so-called T10 elements (P2) consider four additional quadrature points through which the degrees of freedom (DOF) are increased. Thus, T10 elements can behave softer than T4 elements in a simulation. We investigated if this also has an influence on the algorithm's convergence.

\subsubsection{Material parameter}

Finally, the stiffness of the myocardial tissue was altered. In the material law described in section 2.2, the variable $C$ directly relates to the stiffness of the myocardial tissue. To investigate its influence on the convergence, the standard value $C_{0}$ was varied by a factor of $C_{h i g h}=10 \cdot C_{0}$ and $C_{\text {low }}=0.1 \cdot C_{0}$

\section{RESULTS}

An overview of the convergence results for the scenarios introduced in section 2.4 is given in figure 5 . For all charts, the residual norm $q_{r}$ is plotted against the number of iterations $i$.

The convergence of the LV simulations was dominated by the influence of flat areas at the heart base on the displacement of the geometry. Figure 5A shows that when using the LV geometry without any node fixation, the influence of the flat area dominates the convergence result and the algorithm does not converge to a steady state.

To exclude this strong influence for further simulations, different boundary conditions were investigated. A fixation of the basal ring, i.e. a ring of nodes around the lid (figure 4, left) did not improve conver-

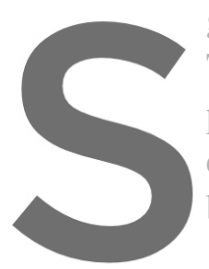
gence. Especially the Thus, all nodes of the 1 prevents a movement of th even if the flat area is stiti basal fixation, other influer
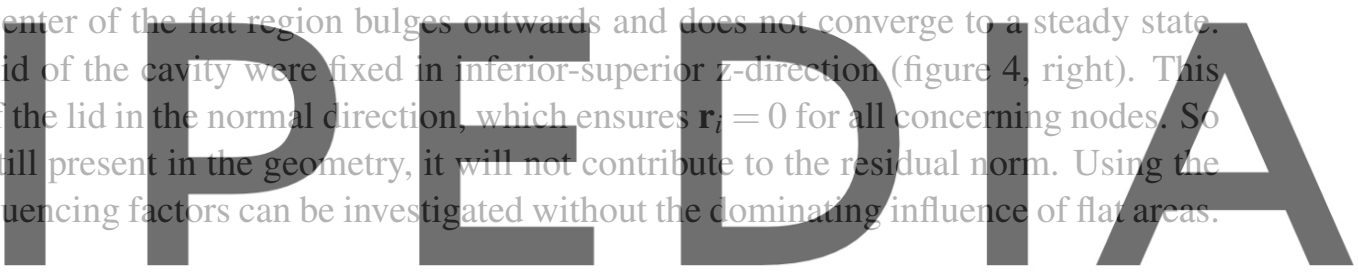

\section{Register for free at hittps//www.scipedia.com to download the version without the watermark}
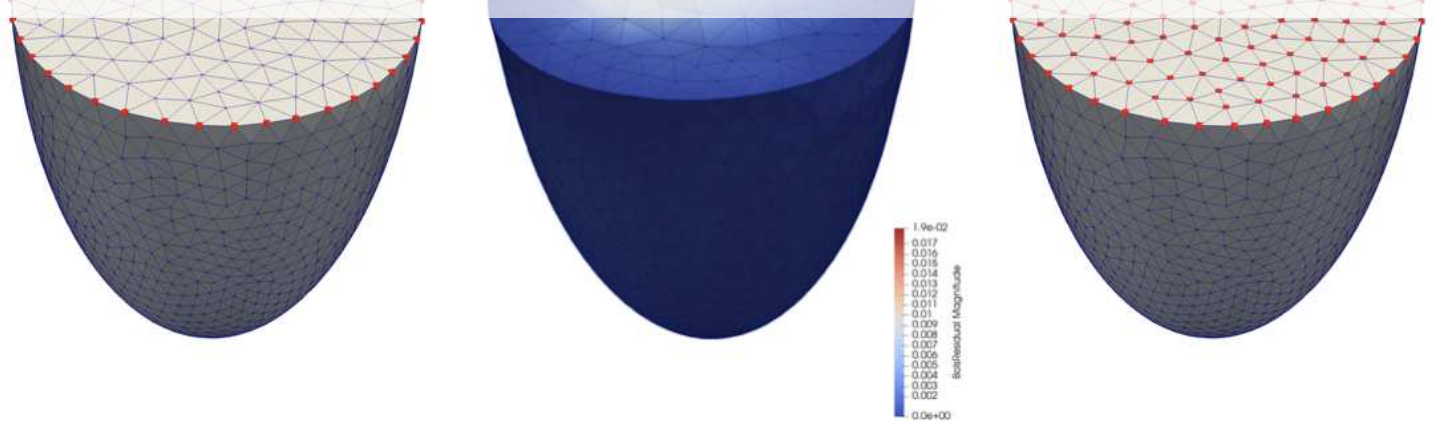

Figure 4: Fixation of basal nodes and resulting Bols residual. Left: Only a ring of nodes (red) encircling the lid is fixed. Middle: Bols residuum for the ring fixation after five iterations. The flat area bulges outwards. Right column: Fixation of all nodes in the lid. 

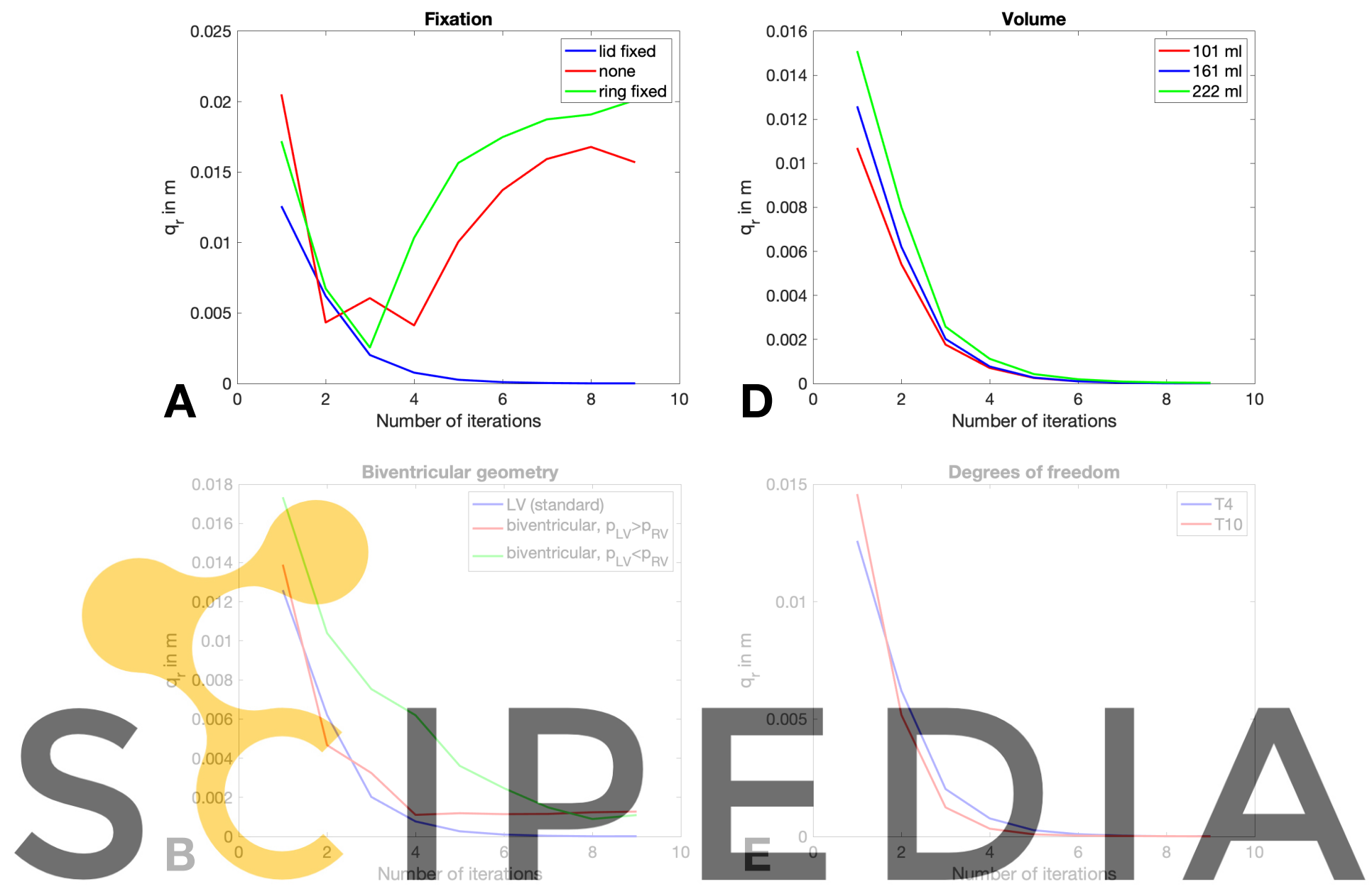

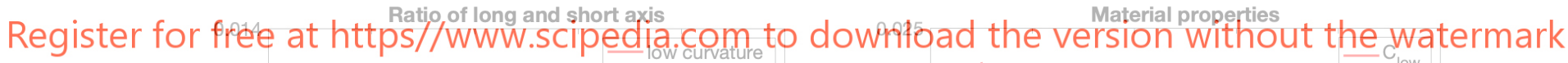
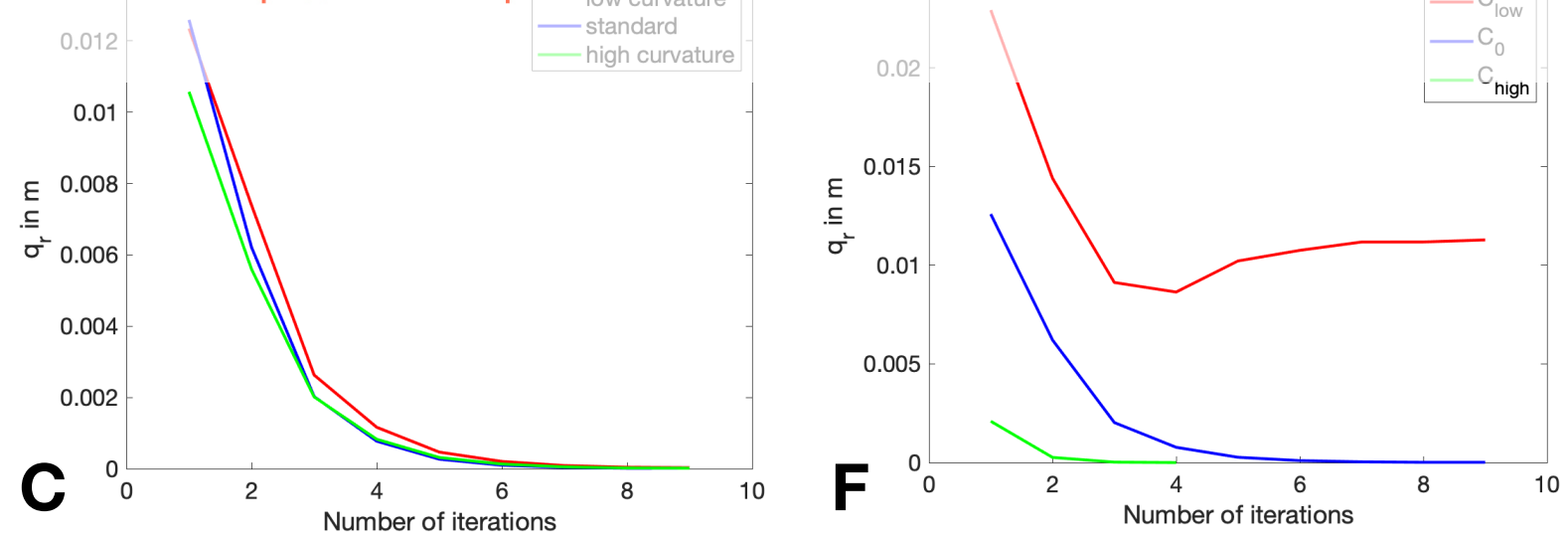

Figure 5: Overview of convergence results. Left Column: Shape alterations for flat, concave and convex areas from top to bottom. Right column: Scenarios with altered ventricular volume, DOF and passive material parameters. 
Figure 5B shows the influence of concave areas. Basis for this analysis was the simulation of the biventricular geometry (figure 1, right). As the RV is attached to the spheroid representing the idealized LV, the septal wall between the two ventricles automatically bulged inside the RV as a concave area. Unloading the biventricular geometry with the end-diastolic pressures denoted in section 2.3 , no influence of the concave area on the algorithm's convergence was observed. This can be explained by the higher pressure in the left ventricle, which prevents the septum from bulging due to RV unloading pressure. The influence of the expanding LV dominated the effect. However, when the two pressures were swapped (i.e., $p_{L V, E D}<p_{R V, E D}$ ), so the LV unloading did no longer stop the septal wall from bulging, the influence of concave shapes became observable. Even if the residual was similar, the convergence rate was lower. The residuals $\mathbf{r}_{i}$ in the final geometry confirmed the concave regions as the cause for the slow convergence.

Altering the ratio between short and long axis did not influence the convergence rate (figure 5C). Also the variation of $\mathrm{LV}$ volume (figure 5D) and the increase of DOF due to the use of quadratic shape functions (T10 elements, figure 5E) did not influence the convergence rate.

Finally, the choice of mechanical material parameters showed a marked effect on the algorithm's convergence and the convergence rate (figure 5F). Higher rigidity of the model increased the convergence rate markedly, potentially due to a more linear dependency between pressure and displacement. Another possible explanation for this behaviour could be the decreased volumetric locking due to smaller deformations of the T4 elements using a stiffer material model $C_{\text {high }}$. Quantitatively, increasing the model stiffness by a factor of ten halved the resulting residual norm after the first iteration of the Bols algorithm. However, decreasing the material parameter to $C_{\text {low }}$ also showed an effect on the convergence of the algorithm. Throughout all iterations,
reached.
4 DISCUSSION
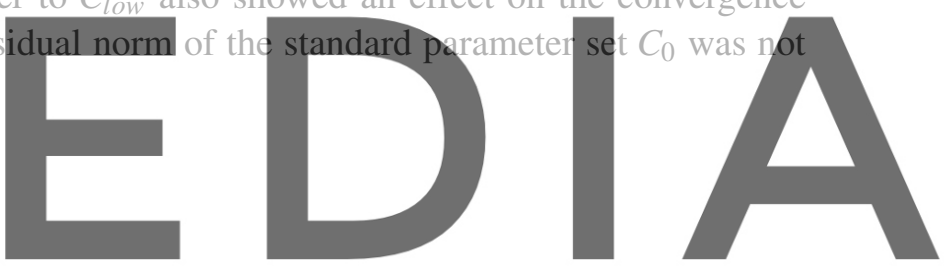

The results of this study highlight a major influence of the material parameter. However, this is not a

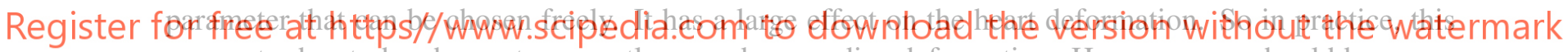
parameter has to be chosen to correctly reproduce cardiac deformation. However, one should be aware that changes of tissue stiffness will have an influence on the unloading procedure convergence.

This study focused on idealized geometries to better understand the causes for low unloading algorithms convergence rates. As good as the simplified geometries are to quantify single effects without confounding factors, the different factors could mutually depend on each other in a whole heart simulation. For example, a flat region modeled using T10 elements could behave different under a varied material parameter.

Secondly this study highlighted the influence of flat and concave areas. Flat areas are another major cause of a slow convergence and can even lead to a failure of the algorithm. However, especially areas in the right ventricle often include flat or even concave areas. Therefore, a locally different cost function for the residual norm could be taken into account in further studies. The weight of problematic regions could be reduced in the cost function to increase algorithm robustness.

A similar adoption of the Bols algorithm was recently suggested in a preprint by Marx et al. [14]. They augmented the standard algorithm by introducing an adaptive displacement factor that scales the residual vector $\mathbf{r}_{i}$ before the backward displacement. Using this adaption, we observed a better convergence in a 
first whole heart simulation using linear elements, namely a smaller residual as well as less oscillations in the residual norm making the algorithm more robust. When applying the adapted algorithm to whole heart model using quadratic T10 elements, we observed better robustness while convergence rate and the residual norm were not improved in preliminary experiments.

A limitation of our study is that the influence of the pericardium surrounding the cardiac chambers was not considered. This is another improvement that could be implemented in further studies, such that during the inflation period, the cardiac tissue has to interact with the pericardium.

In summary, convergence was primarily determined by the rigidity and curvature of the geometry. Factors like volume and DOF did not have a big influence for the presented idealized geometries.

\section{ACKNOWLEDGMENTS}

Funded by the Deutsche Forschungsgemeinschaft (DFG, German Research Foundation) - Project-ID 258734477 - SFB 1173.

\section{REFERENCES}

[1] S. Govindjee, "Computational methods for inverse finite elastostatics," Computer Methods in Applied Mechanics and Engineering, vol. 136, pp. 47-57, 1996.

[2] M. W. Gee, C. Förster, and W. A. Wall, "A computational strategy for prestressing patient-specific biomechanical problems under finite deformation," International Journal for Numerical Methods in Biomedical Engineering, vol. 26, no. 1, pp. 52-72, 2010.
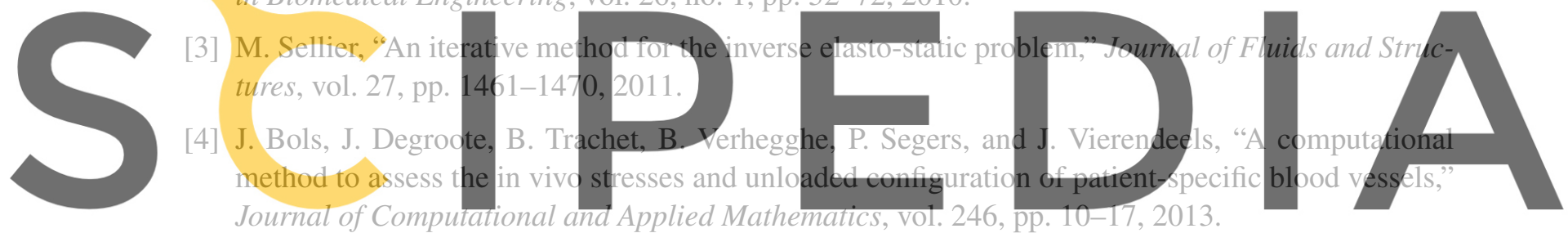

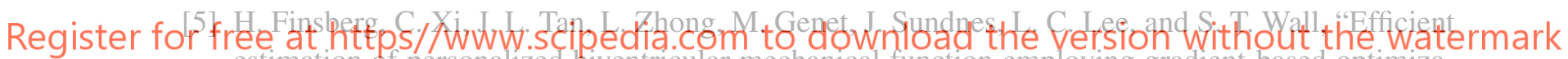
estimation of personalized biventricular mechanical function employing gradient-based optimization," Int. journal for numerical methods in biomedical engineering, vol. 34, no. 7, p. 2982, 2018.

[6] S. Clay, K. Alfakih, A. Radjenovic, T. Jones, J. P. Ridgway, and M. U. Sinvananthan, "Normal range of human left ventricular volumes and mass using steady state free precession MRI in the radial long axis orientation.," Magma (New York, N.Y.), vol. 19, no. 1, pp. 41-5, 2006.

[7] S. Y. Ho, "Anatomy and myoarchitecture of the left ventricular wall in normal and in disease," European Journal of Echocardiography: the Journal of the Working Group on Echocardiography of the European Society of Cardiology, vol. 10, no. 8, pp. 3-7, 2009.

[8] A. Ahmad Bakir, A. Al Abed, M. C. Stevens, N. H. Lovell, and S. Dokos, "A multiphysics biventricular cardiac model: Simulations with a left-ventricular assist device," Frontiers in physiology, vol. 9, p. 1259, 2018.

[9] J. M. Guccione, A. D. McCulloch, and L. K. Waldman, "Passive material properties of intact ventricular myocardium determined from a cylindrical model," Journal of biomechanical engineering, vol. 113, no. 1, pp. 42-55, 1991. 
[10] J. D. Bayer, R. C. Blake, G. Plank, and N. A. Trayanova, "A novel rule-based algorithm for assigning myocardial fiber orientation to computational heart models," Annals of Biomedical Engineering, vol. 40, no. 10, pp. 2243-2254, 2012.

[11] R. E. Peverill, "Understanding preload and preload reserve within the conceptual framework of a limited range of possible left ventricular end-diastolic volumes.," Advances in physiology education, vol. 44, no. 3, pp. 414-422, 2020.

[12] B. Lampert, M. W. Gee, C. Förster, W. A. Wall, M. W. Gee, C. Förster, and W. A. Wall, Right Heart Catheterization. Encyclopedia of Cardiovascular Research and Medicine, Elsevier, 2018.

[13] T. Fritz, C. Wieners, G. Seemann, H. Steen, and O. Dössel, "Simulation of the contraction of the ventricles in a human heart model including atria and pericardium: Finite element analysis of a frictionless contact problem," Biomechanics and Modeling in Mechanobiology, vol. 13, no. 3, pp. 627-641, 2014.

[14] L. Marx, J. A. Niestrawska, M. A. F. Gsell, F. Caforio, G. Plank, and C. M. Augustin, "Efficient identification of myocardial material parameters and the stress-free reference configuration for patient-specific human heart models," Elsevier, 2021.
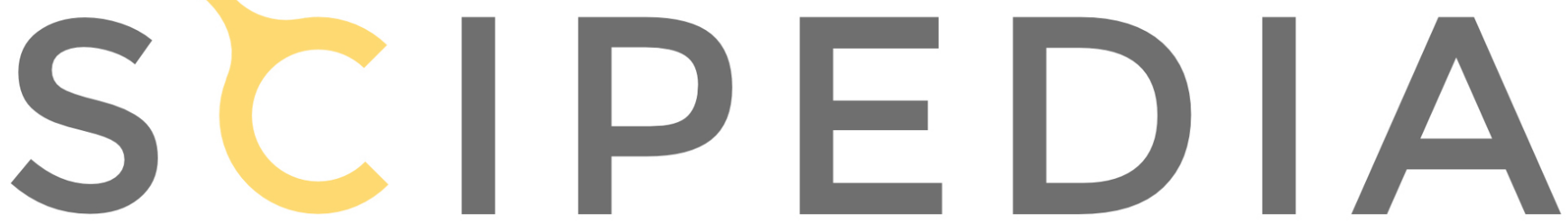

Register for free at https//www.scipedia.com to download the version without the watermark 